AcCepted by ApJ on February 28, 2018

Preprint typeset using $\mathrm{IAT}_{\mathrm{E}} \mathrm{X}$ style emulateapj v. 5/2/11

\title{
DIFFERENT X-RAY SPECTRAL EVOLUTION FOR BLACK HOLE X-RAY BINARIES IN DUAL TRACKS OF RADIO-X-RAY CORRELATION
}

\author{
XiaO-Feng CaO ${ }^{1}$, Qingwen Wu ${ }^{1, \star}$ And Ai-Jun Dong ${ }^{1}$ \\ Accepted by ApJ on February 28, 2018
}

\begin{abstract}
Recently an 'outliers' track of radio-X-ray correlation was found, which is much steeper than the former universal correlation, where dual tracks were speculated to be triggered by different accretion processes. In this work, we test this issue by exploring hard X-ray spectral evolution in four black-hole $\mathrm{X}$-ray binaries (XRBs) with multiple, quasi-simultaneous radio and X-ray observations. Firstly, we find that hard X-ray photon indices, $\Gamma$, are anti- and positively correlated to X-ray fluxes when the $\mathrm{X}$-ray flux, $F_{3-9 \mathrm{keV}}$, is below and above a critical flux, $F_{\mathrm{X} \text {,crit }}$, which are consistent with prediction of advection dominated accretion flow (ADAF) and disk-corona model respectively. Secondly and most importantly, we find that the radio-X-ray correlations are also clearly different when the X-ray fluxes are higher and lower than the critical flux that defined by X-ray spectral evolution. The data points with $F_{3-9 \mathrm{keV}} \gtrsim F_{\mathrm{X} \text {,crit }}$ have a steeper radio-X-ray correlation $\left(F_{\mathrm{X}} \propto F_{\mathrm{R}}^{b}\right.$ and $\left.b \sim 1.1-1.4\right)$, which roughly form the 'outliers' track. However, the data points with anti-correlation of $\Gamma-F_{3-9 \mathrm{keV}}$ either stay in the universal track with $b \sim 0.61$ or stay in transition track (from the universal to 'outliers' tracks or vice versa). Therefore, our results support that the universal and 'outliers' tracks of radio-X-ray correlations are regulated by radiatively inefficient and radiatively efficient accretion model respectively.

Subject headings: X-rays:binaries - black hole physics - accretion, accretion disk - ISM: jets and outflows - radio continuum:stars
\end{abstract}

\section{INTRODUCTION}

Most of Galactic black hole $(\mathrm{BH})$ X-ray bina$\operatorname{ries}(\mathrm{XRBs})$ are transient sources that are detected in X-rays during bright, several-month-long outbursts with typical recurrence periods of many years. During an outburst, the XRB shows different spectral states associated with a q-like pattern in X-ray hardness-intensity diagram (HID). At the beginning and end of the outburst, XRBs are normally observed in low/hard (LH) state, of which the emission is dominated by a powerlaw component extending to $\sim 100 \mathrm{keV}$ with a photon index of $1.5 \lesssim \Gamma \lesssim 2$. XRBs will stay in high/soft (HS) state at high luminosities, where the X-ray spectrum is characterized by a strong thermal black-body emission and a weak power-law component with $\Gamma \gtrsim 2$. Some XRBs also show a steep power-law state with $\Gamma \gtrsim 2.4$, which differs from the HS state in that the power-law component, rather than the disk component, is dominant (Zdziarski 2000; McClintock \& Remillard 2006; Done 2007; Fender \& Belloni 2012; Zhang 2013 for recent reviews).

Different spectral states in XRBs are believed to be triggered by different accretion and/or jet processes. There is little doubt that a cold, optically thick, geometrically thin standard accretion disk (SSD; Shakura \& Sunyaev 1973) captures the basic physical properties of XRBs in HS state. Soft X-ray bumps as observed in HS state can be naturally explained by multitemperature blackbody emission of the SSD. The accretion mode for low/hard (LH) state of XRBs is still a matter of debate. The prevalent accretion model

\footnotetext{
${ }^{1}$ School of Physics, Huazhong University of Science and Technology, Wuhan 430074, China

$\star$ Corresponding author, email: qwwu@hust.edu.cn
}

is a hot, optically thin, geometrically thick advectiondominated accretion flow (ADAF; also called radiatively inefficient accretion flow, RIAF) that has been developed for $\mathrm{BH}$ accreting at low mass accretion rate (e.g., Naravan \& Yi 1994, 1995; Abramowicz et al. 1995; see Ho 2008, Kato et al. 2008, Naravan \& McClintock 2008 and Yuan \& Narayan 2014 for recent reviews). The low density, two-temperature plasma mainly radiates in $\mathrm{X}$ rays, which can well reproduce the observed spectra of XRBs in LH state. We note that there are also some evidences challenge the pure ADAF model for the LH state of XRBs. The broad Fe K emission line (e.g., Miller et al. 2006; Reis et al. 2008; Tomsick et al. 2008) and/or analysis of disk continuum (e.g., Miller et al. 2006; Reis et al. 2010) suggest that SSD may extend to innermost stable circular orbit in some bright hard state of XRBs. For XRBs in a steep power-law state, the accretion process is very unclear, which may be partly caused by the unknown physical mechanism for disk transition (e.g., McClintock \& Remillard 2006).

Numerous studies in the past have shown that hard Xray photon index, $\Gamma$, is correlated with Eddington ratio for both XRBs and AGNs. Wu \& Gu (2008) performed a detailed spectral study for six XRBs in the decay of an outburst and found that $\Gamma$ anti-correlates with $L_{\mathrm{X}} / L_{\mathrm{Edd}}$ ( $L_{\mathrm{Edd}}$ is Eddington luminosity) when $L_{\mathrm{X}} / L_{\mathrm{Edd}}$ is less than a critical value, while they become positively correlated when $L_{\mathrm{X}} / L_{\mathrm{Edd}}$ is higher than this critical value. This phenomena also exist in AGNs, where $\Gamma$ and Eddington ratio are positively correlated in bright AGNs (e.g., quasars and bright Seyferts, Wang et al. 2004; Shemmer et al. 2006; Zhou \& Zhao 2010; Trichas et al. 2013), while the anti-correlations are found in a sample of low-luminosity AGNs (e.g., Gu \& Cao 2009; Constantin et al 2009; Younes et al. 2011). For a single 
AGN, the positive correlation of X-ray photon index and luminosity was also found in several bright sources (e.g., Lamer et al. 2003; Sobolewska \& Papadakis 2009), while the anti-correlation was found in low-luminosity sources (e.g., NGC 7213, Emmanoulopoulos et al. 2012). The anti- and positive correlations between $\Gamma$ and Eddington ratio can be regulated by $\mathrm{ADAF}$ and $\mathrm{SSD} /$ corona respectively as proposed by $\mathrm{Wu} \& \mathrm{Gu}$ (2008). The detailed model calculations on the ADAF and disk/corona do support this scenario (e.g., Cao 2009; You et al. 2012; Qiao \& Liu 2013). From the X-ray spectral evolution, the LH state can be separated into bright hard state and dim hard state, of which the XRBs stay in the positive and anti-correlation of $\Gamma-L_{\mathrm{X}} / L_{\mathrm{Edd}}$ respectively (Wu \& Gu 2008). A small cold disk may exist in some bright hard state of XRBs due to the condensation of hot accretion flow when accretion rate is larger than $\sim 0.01$ Eddington rate, while the inner cold SSD will completely transit to ADAF at lower accretion rate due to the possible evaporation (Liu et al. 2007, 2011).

There is a strong connection between radio and $\mathrm{X}$ ray emission in the $\mathrm{LH}$ state of XRBs. The quasisimultaneous radio and X-ray fluxes of more than ten XRBs roughly follow a universal non-linear correlation of $F_{\mathrm{R}} \propto F_{\mathrm{X}}^{b}(b \sim 0.5-0.7)$ as initially found in LHstate GX 339-4 and V404 Cyg (Hannikainen et al. 1998; Corbel et al. 2003; Gallo et al. 2003), which seems to be maintained down to quiescent state (Gallo et al. 2006). Wu et al. (2013) found that the radio-X-ray relation for a sample of AGNs with $\mathrm{BH}$ mass of $10^{8 \pm 0.4} M_{\odot}$ is roughly similar to that of LH-state XRBs, where the AGN sample with similar $\mathrm{BH}$ mass can be used to simulate the behavior of a single BH XRB in a statistical manner. The quantitative comparison of XRBs and AGNs has made a major step forwards in last ten years with the discovery of a fundamental plane for $\mathrm{BH}$ activity in space of BH mass, radio luminosity and X-ray luminosity (e.g., Merloni et al. 2003; Falcke et al. 2004; Wang et al. 2006; Körding et al. 2006; Li et al. 2008; Yuan et al. 2009; Gültekin et al. 2009; Plotkin et al. 2012). The radio spectrum of XRBs in LH state is usually flat or even inverted with spectral index $\alpha \gtrsim 0\left(F_{\nu} \propto \nu^{\alpha}, F_{\nu}\right.$ is the radio flux at a certain frequency $\nu$ ), which is often taken as the evidence for presence of jets (Fender 2001). The $\mathrm{X}$-ray emission of XRBs is normally thought to originate in the accretion flows (e.g., ADAF, Yuan \& Cui 2005; Narayan \& McClintock 2008). Therefore, the tight correlation of radio and $\mathrm{X}$-ray emission suggests that the jet may be coupled with accretion flow. The physical reasons for the jet formation are still unclear, which possibly include a spinning BH (e.g., Blandford \& Znaiek 1977; Lei et al. 2005; Tchekhovskov et al. 2011; Li \& CaO 2012; Naravan \& McClintock 2012), large-scale magnetic field (e.g., Cao 2011), and/or accretion mode (e.g., Meier 2001) etc. The physical reason for the quenched jet in HS state of XRBs is also still unclear, which may be caused by the decrease of the corona due to the stronger cooling when the SSD is present if the jet is coupled with the hot plasma (ADAF/corona, Wu et al. 2013).

However, Xue \& Cui (2007) found that the radio$\mathrm{X}$-ray correlation in XRBs might not be as universal as previously thought. In the following years, more and more XRBs were found to lie well outside the scatter of the former universal radio-X-ray correlation (e.g., XTE J1650-500, Corbel et al. 2004; XTE J1720-318, Brocksopp et al. 2005; Swift 1753.5-0127, Cadolle Bel et al 2007; Soleri et al. 2010; IGR J174972821, Rodriguez et al. 2007; H1743-322, Jonker et al. 2010; Coriat et al. 2011; XTE J1752-223, Ratti et al. 2012). These 'outliers' roughly form a much steeper 'outliers' track with a correlation slope of $b \sim 1.4$ as initially found in H1743-322 (Coriat et al. 2011). The physical reason for the dual tracks of radio-X-ray correlation is still unclear. Assuming the jet launching and radiation behave identically in both tracks, Coriat et al. (2011) speculated that the universal track and 'outliers' track may be regulated by radiatively inefficient and radiatively efficient accretion flows respectively based on the several simple scalings of accretion and jet.

As addressed above, the hard X-ray spectral evolution can shed light on the accretion modes (e.g., ADAF, disk/corona etc.). In this work, we aim to investigate whether the dual tracks of radio-X-ray correlation are regulated by radiatively inefficient and radiatively efficient accretion flows or not, through exploring their hard $\mathrm{X}$-ray spectral evolution. We present the observation and X-ray data reduction in section 2 . The results are shown in section 3, which are concluded and discussed in section 4.

\section{SAMPLE SELECTION AND X-RAY DATA REDUCTION}

We consider a sample of $\mathrm{BH}$ XRBs in $\mathrm{LH}$ state that have multiple, quasi-simultaneous (e.g., within a day) radio and X-ray observations. In order to explore the X-ray spectral evolution for each XRB, we exclude the sources with only one or several observations. The radio flux densities are obtained from literatures directly, while the $\mathrm{X}$-ray data is selected from $R X T E$ database according to the time of radio observation, and analyzed by ourselves. From these criteria, we select four XRBs (GX 339-4, H 1743-322, Swift J1753.5-0127 and XTE J1752-223). We describe the radio and X-ray data for these sources in more details as follows.

\subsection{Radio data}

For GX 339-4, we select the radio flux densities at 8.6 or $9.0 \mathrm{GHz}$ from Corbel et al. (2013, Table 1), which were observed by Australia Telescope Compact Array (ATCA) during several outbursts from 1997 to 2012. The $8.5 \mathrm{GHz}$ radio data of H1743-322 are selected from Coriat et al. (2011, and references therein), which were observed by ACTA and Very Large Array (VLA) regularly for several outbursts from 2003 to 2010. For Swift J1753.5-0127, Soleri et al. (2010) presented the radio observation with VLA, Westerbork Synthesis Radio Telescope (WRST) and MERLIN at $1.7-8.5 \mathrm{GHz}$ during an outburst from 2005 to 2009. The radio fluxes of three data points observed at $1.7 \mathrm{GHz}$ by MERLIN and four observed by VLA at $4.8 \mathrm{GHz}$ are converted to fluxes at $8.5 \mathrm{GHz}$ assuming a typical radio spectral index of $\alpha=-0.12$ for $\mathrm{LH}$ state of XRBs $\left(F_{\nu} \propto \nu^{-\alpha}\right.$, e.g., Corbel et al. 2013). For XTE J1752-223, we use the radio flux densities at 9.0 $\mathrm{GHz}$ that observed by ATCA during an outburst from 2009 to 2010 (Brocksopp et al. 2013). We list all the radio data in Tables 1-4. In the following analysis, we simply assume the radio flux densities at $8.4-9.0 \mathrm{GHz}$ is 
more or less similar based on the flat spectrum as observed in most LH-state XRBs, which will not affect our results.

\subsection{X-ray spectral analysis}

According to the radio data, we select the quasisimultaneous (within 1 day) X-ray data from $R X T E$ archive. There are 69 observations for GX 339-4 (Table 1), 28 observations for $\mathrm{H}$ 1743-322 (Table 2), 18 observations for Swift J1753.5-0127 (Table 3), and 14 observations for XTE J1752-223 (Table 4). The X-ray data is reduced and analyzed using HEASOFT software following standard steps described in $R X T E$ cookbook 3 .

We extract Proportional Counter Array (PCA, Jahoda et al. 2006) spectra from Standard 2 data, where only top layer of Proportional Counter Unit (PCU) 2 is used since that it is only one operational across all the observations and is the best calibrated detector out of five PCUs. Response matrices are generated and background spectra are created using the latest PCA background model (faint or bright) according to brightness level. We also extract HEXTE (High Energy Timing Experiment, Rothschild et al. 1999) spectra from archive data, where the data from cluster B is considered. However, the Cluster B was also stopped rocking from December 14th 2009.

In order to better exploring the hard X-ray spectral evolution, we analyze the X-ray data in the same energy range for a given XRB. Therefore, we perform a simultaneous fit to the PCA (3-25 keV) and HEXTE (20$200 \mathrm{keV}$ ) spectra in XSPEC for H 1743-322 and Swift J1753.5-0127, where all the data of these two sources are observed before December 2009. However, PCA spectra (3-40 keV) are fitted for GX 339-4 and XTE J1752-223 due to many radio and X-ray data are selected from the outbursts after December 2009. We add a systematic uncertainties of $0.5 \%$ to all spectral channels to account for PCA calibration uncertainties. To normalize between PCA and HEXTE, we multiple the HEXTE model by a constant component. The main aim of the spectral analysis is to derive the X-ray spectral index and unabsorbed X-ray flux. Therefore, we use the model as simple as possible. We use a powerlaw component (powerlaw) and an absorption component (phabs) as starting model, where the hydrogen column density was fixed at $N_{\mathrm{H}}=0.5 \times 10^{22} \mathrm{~cm}^{-2}$ for GX 339-4 (e.g., Kong et al. 2000), $N_{\mathrm{H}}=1.6 \times 10^{22} \mathrm{~cm}^{-2}$ for H 1743-322 (e.g., Capitanio et al 2009), $N_{\mathrm{H}}=1.7 \times$ $10^{21} \mathrm{~cm}^{-2}$ for Swift J1753.5-0127 (e.g., Hiemstra et al. 2009 ), and $N_{\mathrm{H}}=0.46 \times 10^{22} \mathrm{~cm}^{-2}$ for XTE J1752-223 (e.g., Markwardt et al. 2009). A multi-temperature disk blackbody (diskbb), a Gaussian emission line (gaussian) and a high energy cutoff (highecut) will be added if they can improve the fittings substantially (e.g., $\Delta \chi^{2}>0.3$ ). The broken power-law (bknpower) will be used to replace the simple power-law component in some spectral fittings if we cannot get satisfactory fitting result with above models (e.g., $\chi^{2}>2$ ), where the break energy is around $20 \mathrm{keV}$ (e.g., $\mathrm{H}$ 1743-322, McClintock et al. 2009, Swift J1753.5-0127, Soleri et al. e.g., 2010. In addition, we subtract the Galactic ridge emission contam-

\footnotetext{
3 http://heasarc.gsfc.nasa.gov/docs/xte/data_analysis.html
}

ination $6.0 \times 10^{-11} \mathrm{ergs}^{-1} \mathrm{~cm}^{-2}$ (e.g., Coriat et al. 2011) from the unabsorbed flux of $\mathrm{H} 1743-322$ due to the location of $\mathrm{H} 1743-322$ is close to the Galactic plane. The observational date, ID, unabsorbed 3-9 keV X-ray flux, the photon index, fitting results and adopted models are shown in the Tables 1-4.

\section{RESULTS}

We present relations between hard X-ray photon index, $\Gamma$, and 3-9 keV X-ray flux, $F_{3-9 \mathrm{keV}}$, for four sources in top panels of Figures 1-4 respectively, where the

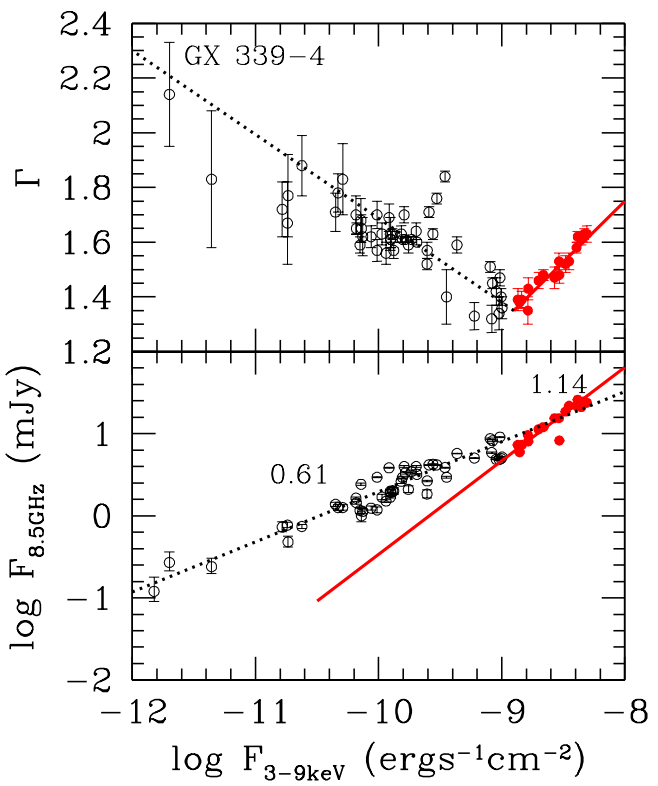

FIG. 1. - Top panel shows the relation between $\mathrm{X}$-ray photon index, $\Gamma$, and unabsorbed 3-9 keV X-ray flux, $F_{3-9 \mathrm{keV}}$, for GX 3394 , where we fit the correlation with a piecewise linear regression (two segments). The dotted and solid lines represent the best fits for the data points in anti- (open circles) and positive (solid circles) correlations respectively. The bottom panel shows the radio-X-ray correlations, where the open and solid circles are defined according to their X-ray spectral evolution in the top panel, where the dotted and solid lines are their best fits (correlation slopes are also shown).

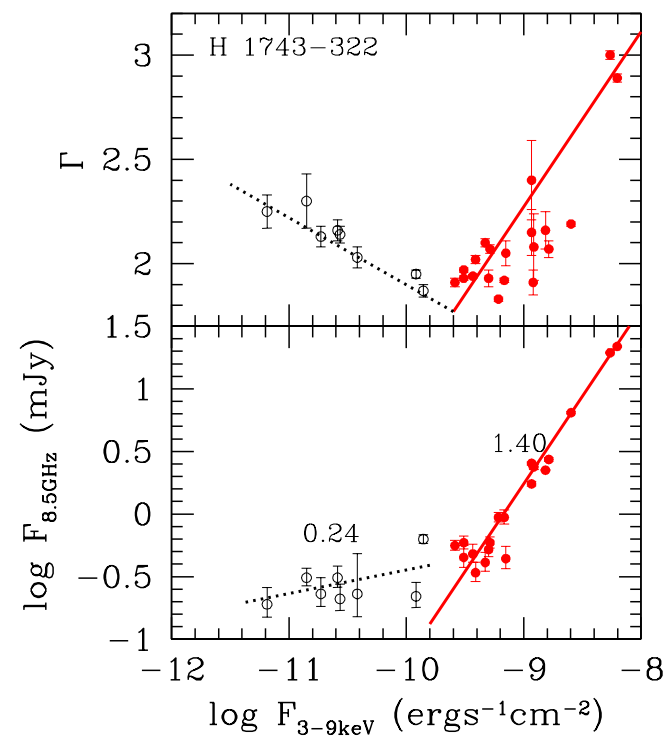

FIG. 2.- The same as Figure 1, but for H 1743-322. 


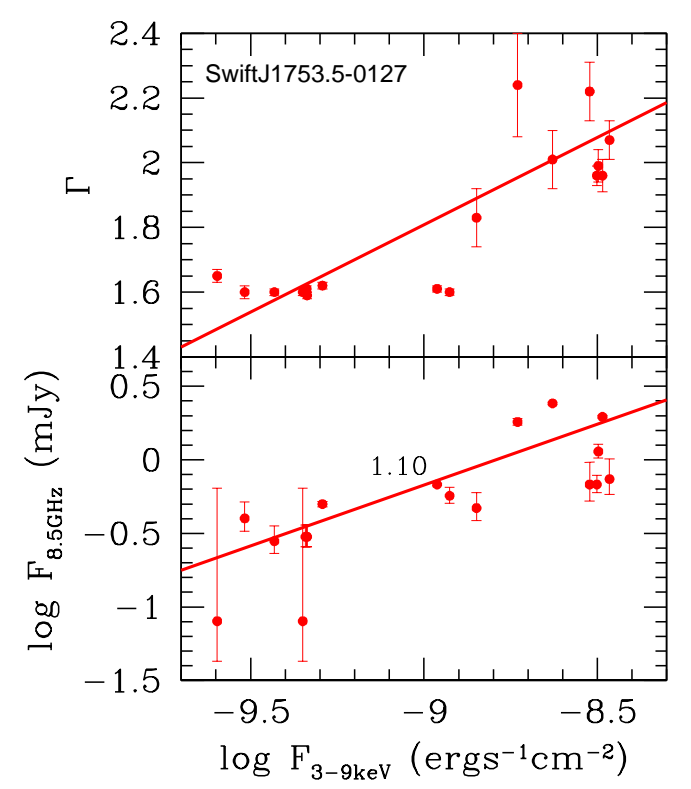

FIG. 3.- The same as Figure 1, but for Swift J1753.5-0127.

photon index at harder band is adopted if it is fitted with a broken power-law model. For GX 339-4 and H 1743-322 (Figures 1 and 2), it can be clearly found that $\Gamma$ is anti-correlated to $F_{3-9 \mathrm{keV}}$ (open circles) when $F_{3-9 \mathrm{keV}}$ is lower than a critical value, $F_{\mathrm{X} \text {,crit }}$, while these two quantities become positively correlated (solid circles) when $F_{3-9 \mathrm{keV}} \gtrsim F_{\mathrm{X} \text {,crit }}$. The positive correlation of $\Gamma-F_{3-9 \mathrm{keV}}$ is evident in Swift J1753.5-0127 as a whole, even though the correlation become flat or a little bit anti-correlated when X-ray flux lower than a critical value (see top panel of Figure 3). For XTE J1752223 , there are some trends in both anti- and positive correlations of $\Gamma-F_{3-9 \mathrm{keV}}$ except that four data points evidently deviate from both anti- and positive correlations (see top panel of Figure 4). We fit the relation of $\Gamma-\log F_{3-9 \mathrm{keV}}$ with a piecewise linear regression (two segments) for each source (top panels in Figures 1-4). Four data points of XTE J1752-223 (with the highest $\mathrm{X}$-ray fluxes) are excluded in the fitting which evidently deviate from both anti- and positive correlations. We list the critical flux (or break point) and its $1 \sigma$ error for the transition of different X-ray spectral evolutions for each XRB in Table 5, where $F_{\mathrm{X} \text {,crit }} \simeq 1.3 \times 10^{-9} \mathrm{ergs}^{-1} \mathrm{~cm}^{-2}$ for GX 339-4 is several times higher than that of other two XRBs $\left(\simeq 2.5 \times 10^{-10} \mathrm{ergs}^{-1} \mathrm{~cm}^{-2}, \mathrm{H} 1743-322\right.$ and XTE J1752-223). In the fitting of Swift J1753.5-0127, only upper limit of critical flux is derived $\left(F_{\mathrm{X}, \text { crit }} \lesssim\right.$ $2.5 \times 10^{-10} \mathrm{ergs}^{-1} \mathrm{~cm}^{-2}$ ), which may be caused by the data points in anti-correlation are not evident or too few.

We find that the radio-X-ray correlation seems to also change along the change of $\Gamma-F_{3-9 \mathrm{keV}}$ correlation (particularly see Figures 1 and 2). Therefore, we divide the data of each source into one (Swift J1753.5-0127) or two groups (GX 339-4 and $\mathrm{H} 1743-322$ ) according to the critical X-ray fluxes as defined by $\Gamma-F_{3-9 \mathrm{keV}}$ correlation. Then we fit the radio-X-ray correlations for each source with a power-law form of $F_{\mathrm{R}}=k F_{\mathrm{X}}^{b}$ for the data points with $F_{3-9 \mathrm{keV}}$ lower or higher than $F_{\mathrm{X} \text {,crit }}$ respectively. In the fittings, the typical flux variations (within one day) $\sigma_{\mathrm{R}}=0.1$ dex in radio band and $\sigma_{\mathrm{X}}=0.15$ dex in X-ray band (e.g., Coriat et al. 2011; Corbel et al. 2013)

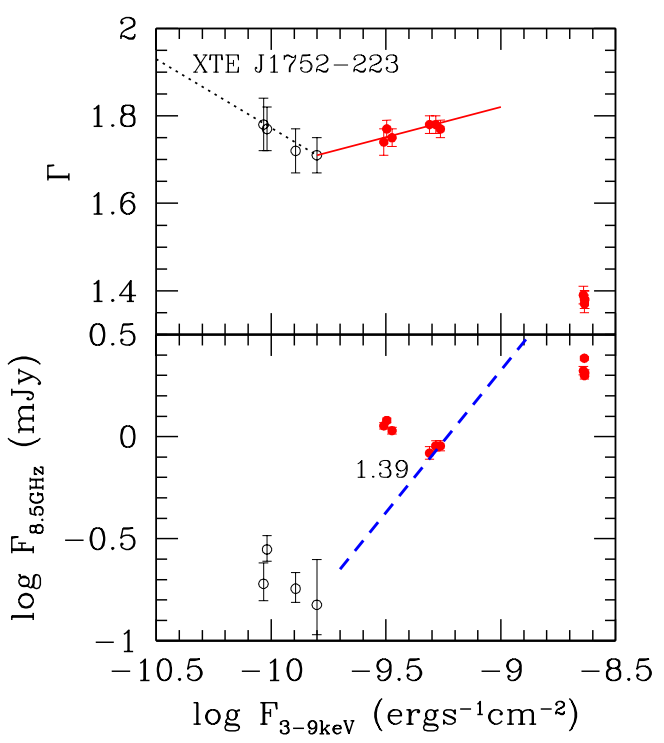

FIG. 4.- The same as Figure 1, but for XTE J1752-223. The dashed line in the bottom panel is not the fitting result for the solid point as defined in the top panel, but the best fit for 17 quasisimultaneous radio/X-ray data points reported in Brocksopp et al. (2013), which are observed from several X-ray satellite and all the selected data points have 3-9 keV X-ray higher than the critical flux defined by X-ray spectral evolution of the top panel.

are assumed to be their flux uncertainties instead of using the observational flux errors. We list the fitting results in Table 5 , where $b_{1}$ and $b_{2}$ represent the correlation slopes for the data points with $F_{3-9 \mathrm{keV}} \lesssim F_{\mathrm{X} \text {,crit }}$ and $F_{3-9 \mathrm{keV}} \gtrsim F_{\mathrm{X}, \text { crit }}$ respectively. We find that the correlation slopes $b_{1}=0.61 \pm 0.03$ for GX $339-4$ and $b_{1}=0.24 \pm 0.10$ for $\mathrm{H} 1743-322$, which are much smaller than the second correlation slopes of $b_{2} \sim 1.1-1.4$. For Swift J1753.5-0127, only the solid points with positive $\Gamma-F_{3-9 \mathrm{keV}}$ correlation are fitted (solid line in bottom panel of Figure 3), and $b_{2}=1.10 \pm 0.16$. Due to insufficient data, the radio-X-ray correlation for XTE $\mathrm{J} 1752-223$ is not fitted with our data. In stead, we select the quasi-simultaneous radio/X-ray data points from Brocksopp et al. (2013) where the X-ray data are reduced from several different X-ray satellites (e.g., Swift, $M A X I$ and/or $R X T E$ ). By excluding the data points with $F_{3-9 \mathrm{keV}} \lesssim F_{\mathrm{X} \text {,crit }}$, we fit the radio-X-ray correlation for 17 data points and find $b_{2}=1.39 \pm 0.19$ (dashed line in bottom panel of Figure 4), where the critical X-ray flux is derived from the X-ray spectral evolution in our work (top panel of Figure 4).

For comparison, we present the correlations of $\Gamma-F_{\mathrm{X}}$ and $F_{\mathrm{R}}-F_{\mathrm{X}}$ for four XRBs in Figure 5. The data points of GX 339-4 with anti-correlation of $\Gamma-F_{\mathrm{X}}$ stay in the former universal track (open squares, bottom panel). All solid points (including GX 339-4) with positive $\Gamma-F_{\mathrm{X}}$ correlation, roughly form the 'outliers' track, even each source seems to follow its own trend. Some data points with anti-correlation of $\Gamma-F_{\mathrm{X}}$ (open circles and triangles) stay in the transition track.

\section{CONCLUSION AND DISCUSSION}

In this work, we analyze the X-ray data from $R X T E$ for a sample of four XRBs with multiple, quasi-simultaneous radio and X-ray observation, and these sources either stay in the universal track or stay in the 'outliers' track as reported in former works. We find that the radio-X- 


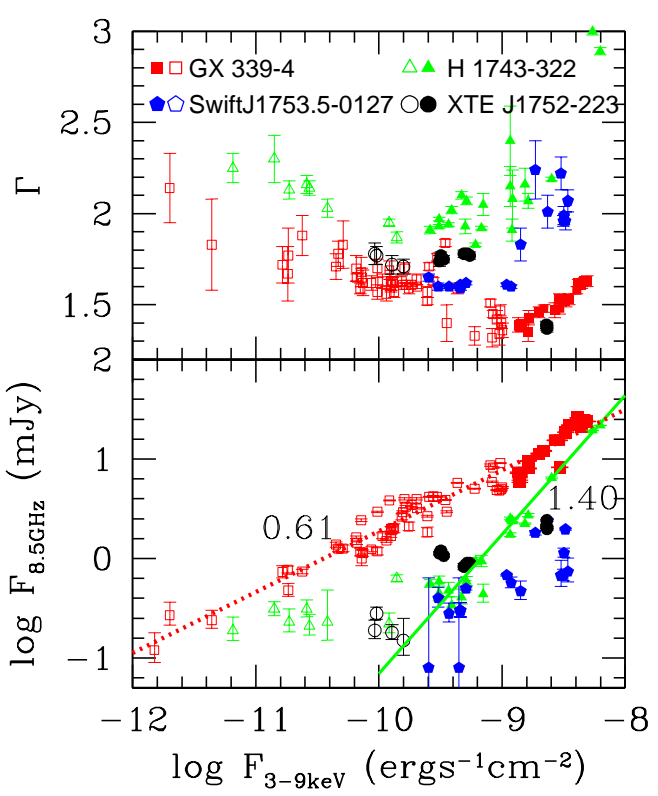

FIG. 5.- The same as Figure 1, but for all sources. For comparison, the best fit of the data points with anti-correlated $\Gamma-F_{3}-9 \mathrm{keV}$ for GX 339-4 (dotted line) and the fit for the data points with positive $\Gamma-F_{3-9 \mathrm{keV}}$ correlation for $\mathrm{H}$ 1743-322 (solid line) are also shown.

ray correlation is tightly correlated to the $\mathrm{X}$-ray spectral evolution. The data points with positive $\Gamma-F_{3-9 \mathrm{keV}}$ correlation normally stay in the steeper 'outliers' track, while the data points with anti-correlation of $\Gamma-F_{3}-9 \mathrm{keV}$ either stay in a shallower universal track or stay in a roughly flat transition track. Both $\Gamma-F_{3-9 \mathrm{keV}}$ correlation and radio-X-ray correlation are changed at similar critical X-ray flux, which support that the different tracks of radio-X-ray correlation should be regulated by different accretion processes if the X-ray emission originate from accretion flows.

The anti- and positive correlations between $\Gamma$ and $F_{3-9 \mathrm{keV}}$, occurring below and above a critical flux, $F_{\mathrm{X} \text {,crit }}$, may be indicative for some 'switch' in radiation mechanism at a certain critical accretion rate. We note that the harder X-ray spectral indices for 17 data points that fitted with a broken power-law are used in investigating of X-ray spectral evolution, which will not affect our main conclusion because both two spectral indices are positively correlated to the X-ray fluxes (see Table 3). Adopting a typical BH mass of $10 M_{\odot}$ (Russell et al. 2013, and references therein) and a distance of $8 \mathrm{kpc}$ (Corbel et al. 2013, and references therein) for these XRBs, we find that the critical Eddington ratio for the transition of anti- and positive $\Gamma-F_{3}-9 \mathrm{keV}$ correlation is $L_{\mathrm{bol}} / L_{\mathrm{Edd}} \sim 1 \%$ for $\mathrm{H} 1743-322$, Swift J1753.50127 and XTE J1752-223, and is 10\% for GX 339-4, where the bolometric luminosity, $L_{\mathrm{bol}}$, is estimated from power-law emission at $0.1-200 \mathrm{keV}$ with the fitted $\mathrm{X}$ ray photon index and flux. The critical Eddington ratio around several percent is roughly consistent with theoretical prediction for transition of ADAF and disk/corona (e.g., Narayan et al. 1998). In ADAF model, the optical depth will increase with increasing of accretion rate, increasing the Compton $y$-parameter, thereby leading to a harder X-ray spectrum, where electron temperature remain roughly unchanged with varying of accretion rate. In disk-corona model, the optical depth of corona will decrease when accretion rate increases due to stronger cooling, which lead to a smaller $y$-parameter and a softer $\mathrm{X}$-ray spectrum. The detailed calculations of ADAF and disk-corona model do predict the anti- and positive correlations of photon index and Eddington ratio as observed in XRBs (e.g., Cao 2009; Qiao \& Liu 2013). Therefore, the different accretion modes may be the physical reason for the change of the X-ray spectral evolution. It should be noted that different values of viscosity parameter $\alpha$ will lead to different critical accretion rates for disk transition (e.g., Liu \& Taam 2009). For a larger $\alpha$, the critical accretion rate for the disk transition will also be large, which may explain why GX 339-4 has a higher critical flux for X-ray spectral evolution than other three XRBs. For XTE J1752-223, there are four data points that evidently deviate from the X-ray spectral evolution of other points (see top panel of Figure 4), which may be caused by the clearly different properties of accretion flow (e.g., magnetic field and or viscosity etc) compared that in other data points. The broadband data and more careful analysis are wished to further investigate their accretion properties, which will be our future work.

The radio-X-ray correlation for the outliers of XRBs have been explored in several XRBs, where the correlation slopes are $\sim 1.1-1.7$ for a certain XRB (Soleri et al. 2010; Rushton et al. 2010; Coriat et al. 2011; Brocksopp et al. 2013). Gallo et al. (2012) reexplored the radio-X-ray correlation for the 'outliers' as a whole, and found that the correlation slope is $\sim 0.98$, where the shallower relation may be caused by the data points in the 'transition' track are also included in their fitting. From the X-ray spectral evolution, the 'outliers' can well separated into two tracks: the 'outliers' track with only positive correlation of $\Gamma-F_{3-9 \mathrm{keV}}$ and the transition track with anti-correlation of $\Gamma-F_{3-9 \mathrm{keV}}$. Excluding the data points in the transition track, the radio-X-ray correlation of the 'outliers' track will become steeper, and this is the reason why our correlation slopes are larger than those reported in former works. It is interesting to note that the bright hard state of GX 339-4 with positive correlation of $\Gamma-F_{3}-9 \mathrm{keV}$ also shows a much steeper radio-X-ray correlation $(\sim 1.1)$ than the universal one. Therefore, all these data points may also belong to the 'outliers' track even this source was divided into the universal track in former works (Coriat et al. 2011; Corbel et al. 2013). The correlation slopes are different for different 'outliers' of XRBs, which may be caused by the different properties of accreting matter (e.g., magnetic field etc.). Due to the different trends of radio-Xray correlation in each $\mathrm{XRB}$, we don't fit the XRBs in the 'outliers' track as a whole in $F_{\mathrm{R}}-F_{\mathrm{X}}$ relation. The luminosity-luminosity relation and/or Eddington-scaled luminosity-luminosity relation for a sample of XRBs may be more intrinsic, and, however, the distances and $\mathrm{BH}$ masses are still very uncertain for these several XRBs.

Coriat et al. (2011) proposed that different radio-Xray correlations are possibly regulated by different accretion processes, which is supported by our results on the hard X-ray spectral evolution. For a classical jet, the jet power, $P_{\text {iet }}$, is a fraction of accretion power and $P_{\text {iet }} \propto \dot{M}$ (e.g., Falcke \& Biermann 1995; Heinz \& Sunveav 2003). In the optically thick jet, the radio emission and jet power follow a scaling of $L_{\mathrm{R}} \propto P_{\text {jet }}^{\sim 1.4}$ (e.g., Heinz \& Sunyeav 
2003; Coriat et al. 2011). The radiatively inefficient ADAF is expected to produce X-ray emission with $L_{\mathrm{X}} \propto$ $\dot{M}^{q}$ and $q \sim 2.0$ (e.g., Merloni et al. 2003; Yuan \& Cui 2005; Wu \& CaO 2006). Therefore, the ADAF model can not only explain the anti-correlation of $\Gamma-F_{3-9 \mathrm{keV}}$ (e.g., Qiao \& Liu 2013), but can also reproduce the observed universal correlation of $L_{\mathrm{R}} \propto L_{\mathrm{X}}^{\sim 0.7}$. For a magnetically heated corona above and below SSD, $q \simeq 1$ for a gas pressure dominated SSD, while $q<1$ for a radiation pressure dominated SSD (e.g., Merloni \& Fabian 2002). We expect $L_{\mathrm{R}} \propto L_{\mathrm{X}} \tilde{\mathrm{N}}^{1.4}$, which is supported by the detailed calculations based on the jet formation from the diskcorona system (Huang et al. 2014). Therefore, the diskcorona model can explain both the steeper radio-X-ray correlation and the positive $\Gamma-F_{3-9 \mathrm{keV}}$ relation simultaneously. From our results, the dual tracks of radio-X-ray correlation are most possibly regulated by different accretion processes. The 'radio quiet outliers' compared those sources in the universal track are caused by 'X-ray loud' due to the increasing of the radiative efficiency as change of accretion mode. The transition track may be regulated by a transition state of accretion flow. The radiative efficiency will increase very fast at a critical ac- cretion rate (e.g., from low radiative efficiency of ADAF to high radiative efficiency of disk-corona). If this is the case, we expect the X-ray luminosity will increase much faster than the radio luminosity if the accretion rate approach to the critical rate for disk transition, which can roughly explain the flat transition track. However, the physical reason for disk transition is still unclear, and the possible models include truncated SSD-ADAF (e.g., Lu et al. 2004), clumpy-ADAF (e.g., Wang et al. 2012), magnetically dominated accretion flow (e.g., Oda et al. 2012) and luminous hot accretion flow (e.g., Yuan 2001; Xie \& Yuan 2012), etc. These transitional disks are more or less ADAF-like, so the anti-correlated $\Gamma-F_{3-9 \mathrm{keV}}$ will be also expected, and the detailed spectral calculation is beyond of this work.

\section{ACKNOWLEDGEMENTS}

We thanks Xinwu Cao and members of HUST astrophysics group for many useful discussions and comments. This work is supported by the NSFC (grants 11303010, 11103003 and 11133005) and New Century Excellent Talents in University (NCET-13-0238).

\section{REFERENCES}

Abramowicz, M. A., Chen, X., Kato, S., et al. 1995, ApJ, 438, L37 Blandford, R. D., \& Znajek, R. L. 1977, MNRAS, 179, 433

Brocksopp, C.; Corbel, S., Tzioumis, A.,Broderick, J. W., Rodriguez, J., Yang, J., Fender, R. P., \& Paragi, Z. 2013, MNRAS, 432, 931

Brocksopp, C., Corbel, S., Fender, R. P., Rupen, M., Sault, R., Tingay, S. J., Hannikainen, D., \& O'Brien, K. 2005, MNRAS, 356,125

Cadolle, Bel M., Ribó M., Rodriguez, J., et al., 2007, ApJ, 659, 549

Cao, X. 2009, MNRAS, 394, 207

Cao, X. 2011, ApJ, 737, 94

Capitanio, F., Belloni, T., Del Santo, M., \& Ubertini, P. 2009, MNRAS, 398, 1194

Constantin, A., Green, P., Aldcroft, T., Kim, D.-W., Haggard, D., Barkhouse, W., \& Anderson, S. F. 2009, ApJ, 705, 1336

Corbel S., Nowak M. A., Fender R. P., et al. 2003, A\&A, 400, 1007

Corbel S., Fender R. P., Tomsick J. A., et al. 2004, ApJ, 617, 1272

Corbel S., Coriat M., Brocksopp C., et al. 2013, MNRAS, 428, 2500

Coriat M., Corbel S., Prat L., et al. 2011, MNRAS, 414, 677

Done, C. Gierlinski, M., Kubota, A. 2007, A\&ARv, 15, 1

Done C., Trigo M. D. 2010, MNRAS, 407, 2287

Emmanoulopoulos, D., Papadakis, I. E., McHardy, I. M., Arevalo, P., Calvelo, D. E., \& Uttley, P. 2012, MNRAS, 424, 1327

Falcke, H., Biermann, P. L. 1995, A\&A, 293, 665

Falcke, H., Körding, E., Markoff, S., 2004, A\&A, 414, 895

Fender, R., \& Belloni, T. 2012, Science, 337, 540

Fender, R. P. 2001, MNRAS, 322, 31

Gallo, E., Fender, R. P., Pooley, G. G. 2003, MNRAS, 344, 60

Gallo, E., Fender, R. P., Miller-Jones, J. C. A., Merloni, A., Jonker, P. G., Heinz, S., Maccarone, T. J., van der Klis, M. 2006, MNRAS, 370, 1351

Gallo, E., Miller, B. P., Fender, R. 2012, MNRAS, 423, 590

Gültekin, M., Cackett, E. M., Miller, J. M., et al. 2009, ApJ, 706, 404

Gu, M., Cao, X. 2009, MNRAS, 399, 349

Hannikainen, D. C., Hunstead, R.-W., Campbell-Wilson, D., Sood,R. K. 1998, A\&A, 337, 460

Heinz, S., Sunyeav, R., 2003, MNRAS, 343, L59

Hiemstra, B., Soleri, P., Mendez, M., Belloni, T., Mostafa, R., \& Wijnands, R. 2009, MNRAS, 394, 2080

Ho, L. C. 2008 , ARA\&A, 46, 475

Huang, C.-Y., Wu, Q. \& Wang, D.-X. 2014, MNRAS, in press

Jahoda, K., et al. 2006, ApJS, 163, 401
Jonker, P. G., Miller-Jones, J., Homan, J. 2010, MNRAS, 401, 1255

Kato, S., Fukue, J., Mineshige, S. 2008, Black Hole Accretion Disks, Kyoto University Press

Kording, E. G., Jester, S., \& Fender, R. 2006, MNRAS, 372, 1366

Kong, A. K. H., Kuulkers, E., Charles, P. A., \& Homer, L. 2000, MNRAS, 312, 49

Lamer, G., et al. 2003, MNRAS, 338, 323

Lei, W.-H., Wang, D.-X., \& Ma, R.-Y. 2005, ApJ, 619, 420

Li, S.-L., \& Cao, X. 2012, ApJ, 753, 24

Li, Z.-Y., Wu, X.-B., Wang, R. 2008, ApJ, 688, 826

Liu, B.-F., Taam, R. E., Meyer-Hofmeister, E., Meyer, F. 2007, ApJ, 671, 695

Liu, B. F., Done, C., \& Taam, R. E. 2011, ApJ, 726, 10

Liu, B.-F., Taam, R. E. 2009, ApJ, 707, 233

Lu, J.-F., Lin, Y.-Q., Gu, W.-M. 2004, ApJ, 602, 37

Markoff, S., \& Nowak, M. A. 2005, ApJ, 635, 1203

Markwardt, C. B., et al. 2009, ATel, 2258, 1

McClintock, J. E., Remillard, R. A., Rupen, M. P., Torres, M. A. P., Steeghs, D., Levine, A. M., \& Orosz, J. A. 2009, ApJ, 698, 1398

McClintock, J. E., Remillard, R. A., 2006, in Lewin, van der Klis, eds, Compact Stellar X-ray Sources. Cambridge Univ. Press, Cambridge, p. 157

Meier, D. L. 2001, ApJ, 548, L9

Merloni, A. \& Fabian, A. C. 2002, 332, 165

Merloni, A., Heinz, S., Matteo, T. D. 2003, MNRAS, 345, 1057

Miller, J. M., Homan, J., Steeghs, D., et al. 2006, ApJ, 653, 525

Narayan, R., Yi, I. 1994, ApJ, 428, L13

Narayan, R., Yi, I. 1995, ApJ, 444, 231

Narayan, R., Mahadevan, R., \& Quataert, E. 1998, in The Theory of Black Hole Accretion Discs, ed. M. A. Abramowicz, G. Bjornsson, \& J. E. Pringle (Cambridge: Cambridge Univ. Press), 148

Narayan, R., \& McClintock, J. E. 2008, NewAR, 51, 733

Narayan, R., \& McClintock, J. E. 2012, MNRAS, 419, 69

Oda, H., Machida, M., Nakamura, K. E., Matsumoto, R., Narayan, R. 2012, PASJ, 64, 15

Plotkin, R. M., Markoff, S., Kelly, B. C., Kording, E., Anderson, S. F. 2012, MNRAS, 419, 267

Plant, D. S., Fender, R. P., Ponti, G., Munoz-Darias, T., Coriat, M. 2013, submitted to MNRAS, arXiv:1309.4781

Qiao, E., Liu, B. F. 2013, ApJ, 764, 2

Ratti, E. M., Jonker P. G., Miller-Jones J. A. C., et al. 2012, MNRAS, 423, 2656 
Reis R. C., Fabian A. C., Ross R. R., et al. 2008, MNRAS, 387, 1489

Reis, R. C., Fabian, A. C., Miller, J. M. 2010, MNRAS, 402, 836

Rodriguez, J., et al. 2007, ApJ, 655, 97

Rothschild, R. E., et al. 1999, ApJ, 496, 538

Rushton, A., Spencer, R., Fender, R., \& Pooley, G. 2010, A\&A, 524, 29

Russell, D. M., Maccarone, T. J., Kording, E. G., \& Homan, J. 2007, MNRAS, 379, 1401

Russell, D. M.; Gallo, E.; Fender, R. P. 2013,MNRAS,431,405

Shakura, N. I., Sunyaev, R. A. 1973, A\&A, 24, 337

Shemmer, O., Brandt, W. N., Netzer, H., Maiolino, R., Kaspi, S. 2006, ApJ, 646, 29

Sobolewska, M. A., Papadakis, I. E. 2009, MNRAS, 399, 1597

Soleri, P., Fender, R., Tudose, V., et al., 2010, MNRAS, 406, 1471

Spruit, H. C. 2010, Lecture Notes in Physics, 794, 233

Tchekhovskoy, A., Narayan, R., \& McKinney, J. C. 2011, MNRAS, 418, 79

Tomsick, J. A., Kalemci, E., Kaaret, P., et al., 2008, ApJ, 680, 593

Trichas, M., et al. 2013, ApJ, 778, 188
Wang, J.-M., Cheng, C., \& Li, Y.-R. 2012, ApJ, 748, 147

Wang, J.-M., Watarai, K.-Y,. Mineshige, S. 2004, ApJ, 607, 107

Wang, R., Wu, X.-B., Kong M.-Z. 2006, ApJ, 645, 890

Wu, Q., Gu, M. 2008, ApJ, 682, 212

Wu, Q., Cao, X., Ho, L. C., Wang, D.-X. 2013, ApJ, 770, 31

Wu Q., Cao X. 2006, PASP, 118, 1098

Xie, F.-G., \& Yuan, F. 2012, MNRAS, 427, 1580

Xue, Y. Q., Cui, W. 2007, A\&A, 466, 1053

You, B., Cao, X., Yuan, Y.-F. 2012, ApJ, 761, 109

Younes, G., Porquet, D., Sabra, B., \& Reeves, J. N. 2011, A\&A, 530,149

Yuan, F., \& Narayan, R. 2014, ARA\&A, in press

Yuan, F., Yu Z., Ho, L. C. 2009, ApJ, 703, 1034

Yuan, F., Cui, W. 2005, ApJ, 629, 408

Yuan, F. 2001, MNRAS, 324, 119

Zdziarski, A. A. 2000, in Highly Energetic Physical Processes,

Procs. IAU Symposium $\sharp 195$, eds. C. H. Martens, S. Tsuruta and M. A. Weber, ASP, 153-170

Zhang, S.-N. 2013, Frontiers of Physics (arXiv:1302.5485)

Zhou, X.-L., \& Zhao, Y.-H. 2010, ApJ, 720, 206 
Cao et al.

TABLE 1 Summary of data for GX 339-4.

\begin{tabular}{|c|c|c|c|c|c|c|}
\hline Date & Obs. Id & $\begin{array}{c}F_{\mathrm{R}} \\
8.6 \text { or } 9.0 \mathrm{GHz} \\
\text { mJy } \\
\end{array}$ & $\begin{array}{c}F_{\mathrm{X}} \\
3-9 \mathrm{keV} \\
10^{-11} \mathrm{erg} / \mathrm{s} / \mathrm{cm}^{2}\end{array}$ & $\Gamma$ & $\chi_{\nu}^{2}(\mathrm{dof})$ & Model $^{a}$ \\
\hline 1997-02-03 & 20181-01-01-01 & $9.10 \pm 0.10$ & $96.70_{-0.30}^{+0.30}$ & $1.47_{-0.03}^{+0.02}$ & $0.86(65)$ & diskbb+gau+pow \\
\hline 1997-02-10 & 20181-01-02-00 & $8.20 \pm 0.20$ & $84.26_{-0.24}^{+0.24}$ & $1.45_{-0.02}^{+0.02}$ & $1.03(65)$ & diskbb+gau+pow \\
\hline $1997-02-17$ & 20181-01-03-00 & $8.70 \pm 0.20$ & $80.68_{-0.23}^{+0.23}$ & $1.51_{-0.02}^{+0.02}$ & $0.90(65)$ & diskbb + gau + pow \\
\hline 1999-03-03 & 40108-01-04-00 & $5.74 \pm 0.06$ & $43.32_{-0.16}^{+0.16}$ & $1.59_{-0.03}^{+0.0 \overline{3}}$ & $1.31(66)$ & diskbb + gau + pow \\
\hline 1999-04-22 & 40108-02-02-00 & $3.20 \pm 0.06$ & $20.44_{-0.08}^{+0.09}$ & $1.60_{-0.01}^{+0.01}$ & $1.27(61)$ & gau + pow \\
\hline 1999-05-14 & 40108-02-03-00 & $1.44 \pm 0.06$ & $6.60_{-0.06}^{+0.06}$ & $1.65_{-0.02}^{+0.02}$ & $0.89(63)$ & pow \\
\hline 1999-06-25 & 40105-02-02-00 & $0.24 \pm 0.05$ & $0.44_{-0.07}^{+0.07}$ & $1.83_{-0.25}^{+0.26}$ & $0.49(63)$ & pow \\
\hline 1999-07-07 & 40108-03-01-00 & $0.12 \pm 0.04$ & $0.15_{-0.03}^{+0.03}$ & $2.18_{-0.20}^{+0.21}$ & $0.50(63)$ & pow \\
\hline 1999-08-17 & 40108-03-03-00 & $0.27 \pm 0.07$ & $0.20_{-0.04}^{+0.04}$ & $2.14_{-0.19}^{+0.20}$ & $0.58(63)$ & pow \\
\hline 2002-04-03 & 70109-01-02-00 & $5.95 \pm 0.15$ & $139.76_{-0.42}^{+0.42}$ & $1.38_{-0.02}^{+0.02}$ & $0.90(59)$ & diskbb + gau + pow \\
\hline 2002-04-07 & 70109-01-01-00 & $8.27 \pm 0.07$ & $293.89_{-1.09}^{+1.09}$ & $1.48_{-0.03}^{+0.02}$ & $1.02(59)$ & diskbb + gau + pow \\
\hline 2003-05-25 & 80102-04-07-00 & $0.77 \pm 0.06$ & $1.82_{-0.13}^{+0.13}$ & $1.67_{-0.15}^{+0.15}$ & $0.61(64)$ & pow \\
\hline 2004-02-13 & $80102-04-57-01$ & $1.13 \pm 0.08$ & $7.44_{-0.23}^{+0.23}$ & $1.62_{-0.07}^{+0.07}$ & $0.72(64)$ & pow \\
\hline 2004-02-24 & 80102-04-58-01 & $1.84 \pm 0.20$ & $24.74_{-0.29}^{+0.29}$ & $1.52_{-0.02}^{+0.02}$ & $0.77(64)$ & pow \\
\hline 2004-03-16 & 90118-01-05-00 & $4.88 \pm 0.06$ & $90.21_{-0.41}^{+0.41}$ & $1.42_{-0.05}^{+0.03}$ & $0.97(60)$ & diskbb+gau+pow \\
\hline 2004-03-17 & 90118-01-06-00 & $4.84 \pm 0.11$ & $95.23_{-0.48}^{+0.50}$ & $1.34_{-0.06}^{+0.05}$ & $0.66(60)$ & diskbb+gau+pow \\
\hline 2004-03-18 & 90118-01-07-00 & $4.98 \pm 0.11$ & $98.89_{-0.32}^{+0.32}$ & $1.40_{-0.03}^{+0.02}$ & $1.14(60)$ & diskbb + gau + pow \\
\hline 2004-03-19 & 80132-01-15-00 & $5.20 \pm 0.10$ & $100.62_{-0.38}^{+0.38}$ & $1.36_{-0.04}^{+0.04}$ & $0.85(60)$ & diskbb+gau+pow \\
\hline 2005-04-24 & 90704-01-13-01 & $4.23 \pm 0.08$ & $27.65_{-0.27}^{+0.27}$ & $1.63_{-0.02}^{+0.02}$ & $0.98(64)$ & pow \\
\hline 2005-04-28 & 91095-08-06-00 & $3.46 \pm 0.13$ & $18.47_{-0.11}^{+0.11}$ & $1.61_{-0.01}^{+0.01}$ & $0.99(62)$ & gau+pow \\
\hline 2005-04-29 & 91095-08-07-00 & $3.32 \pm 0.10$ & $16.49_{-0.10}^{+0.10}$ & $1.61_{-0.01}^{+0.01}$ & $0.96(62)$ & gau + pow \\
\hline 2005-04-30 & $91095-08-08-00$ & $2.94 \pm 0.07$ & $15.63_{-0.10}^{+0.10}$ & $1.61_{-0.01}^{+0.01}$ & $1.17(64)$ & pow \\
\hline 2005-05-02 & 90165-01-01-02 & $1.92 \pm 0.14$ & $12.57_{-0.16}^{+0.16}$ & $1.61_{-0.03}^{+0.03}$ & $1.07(64)$ & pow \\
\hline 2005-05-04 & 91105-04-18-00 & $1.99 \pm 0.10$ & $12.46_{-0.32}^{+0.32}$ & $1.63_{-0.06}^{+0.06}$ & $0.80(64)$ & pow \\
\hline 2005-05-06 & 90704-01-14-00 & $1.69 \pm 0.12$ & $10.64_{-0.18}^{+0.18}$ & $1.63_{-0.04}^{+0.04}$ & $0.60(64)$ & pow \\
\hline 2005-05-12 & 90704-01-14-02 & $1.00 \pm 0.18$ & $7.26_{-0.17}^{+0.17}$ & $1.65_{-0.05}^{+0.05}$ & $1.00(64)$ & pow \\
\hline 2007-06-06 & 92704-03-25-00 & $2.63 \pm 0.18$ & $15.28_{-0.18}^{+0.18}$ & $1.63_{-0.03}^{+0.03}$ & $1.04(64)$ & pow \\
\hline 2007-06-11 & 92704-03-29-00 & $2.01 \pm 0.15$ & $12.98_{-0.13}^{+0.13}$ & $1.63_{-0.02}^{+0.02}$ & $1.31(64)$ & pow \\
\hline 2007-06-25 & 92704-03-38-00 & $1.69 \pm 0.05$ & $12.51_{-0.15}^{+0.15}$ & $1.61_{-0.03}^{+0.03}$ & $1.03(64)$ & pow \\
\hline 2007-06-29 & $92704-03-42-00$ & $2.00 \pm 0.20$ & $13.28_{-0.18}^{+0.18}$ & $1.57_{-0.03}^{+0.03}$ & $0.92(64)$ & pow \\
\hline 2007-07-04 & 92704-03-44-01 & $2.10 \pm 0.20$ & $17.46_{-0.25}^{+0.25}$ & $1.59_{-0.03}^{+0.03}$ & $0.79(64)$ & pow \\
\hline 2007-07-13 & $92704-03-47-00$ & $2.66 \pm 0.05$ & $24.82_{-0.34}^{+0.34}$ & $1.57_{-0.03}^{+0.03}$ & $0.68(64)$ & pow \\
\hline $2007-08-23$ & 93409-01-05-03 & $2.95 \pm 0.07$ & $35.63_{-0.25}^{+0.25}$ & $1.40_{-0.10}^{+0.08}$ & $0.95(60)$ & diskbb + gau + pow \\
\hline $2007-12-27$ & 93409-01-20-00 & $0.48 \pm 0.07$ & $1.84_{-0.13}^{+0.13}$ & $1.77_{-0.15}^{+0.16}$ & $0.61(64)$ & pow \\
\hline $2008-06-26$ & 93076-08-01-00 & $1.16 \pm 0.10$ & $7.08_{-0.10}^{+0.10}$ & $1.59_{-0.03}^{+0.03}$ & $0.97(64)$ & pow \\
\hline 2008-07-05 & 93076-08-03-00 & $1.24 \pm 0.07$ & $8.68_{-0.15}^{+0.15}$ & $1.62_{-0.04}^{+0.04}$ & $0.70(64)$ & pow \\
\hline 2008-07-16 & 93076-08-05-05 & $1.51 \pm 0.06$ & $11.58_{-0.23}^{+0.23}$ & $1.56_{-0.04}^{+0.04}$ & $0.93(64)$ & pow \\
\hline 2008-08-18 & 93108-01-01-02 & $1.18 \pm 0.10$ & $9.72_{-0.16}^{+0.16}$ & $1.57_{-0.04}^{+0.04}$ & $0.67(64)$ & pow \\
\hline $2008-10-10$ & $93702-04-03-00$ & $0.73 \pm 0.10$ & $1.65_{-0.08}^{+0.08}$ & $\begin{array}{l}1.72_{-0.10}^{+0.10} \\
\end{array}$ & $0.49(64)$ & pow \\
\hline 2010-01-21 & 95409-01-02-02 & $5.05 \pm 0.05$ & $59.93_{-0.29}^{+0.29}$ & $1.33_{-0.05}^{+0.05}$ & $0.58(60)$ & diskbb+gau + pow \\
\hline 2010-02-12 & 95409-01-06-00 & $5.90 \pm 0.10$ & $82.83_{-0.37}^{+0.37}$ & $1.32_{-0.05}^{+0.04}$ & $0.56(60)$ & diskbb + gau + pow \\
\hline 2010-03-02 & 95409-01-08-02 & $7.30 \pm 0.10$ & $134.94_{-0.57}^{+0.60}$ & $1.39_{-0.04}^{+0.03}$ & $1.12(60)$ & diskbb + gau + pow \\
\hline 2010-03-04 & 95409-01-08-03 & $7.30 \pm 0.10$ & $145.24_{-0.51}^{+0.51}$ & $1.39_{-0.03}^{+0.02}$ & $0.81(60)$ & diskbb + gau + pow \\
\hline 2010-03-06 & 95409-01-09-06 & $9.60 \pm 0.05$ & $163.27_{-0.74}^{+0.74}$ & $1.35_{-0.05}^{+0.05}$ & $0.88(60)$ & diskbb + gau + pow \\
\hline 2010-03-07 & 95409-01-09-01 & $8.05 \pm 0.10$ & $164.11_{-0.86}^{+0.86}$ & $1.43_{-0.04}^{+0.04}$ & $0.86(60)$ & diskbb + gau + pow \\
\hline 2010-03-14 & 95409-01-10-02 & $11.32 \pm 0.10$ & $199.22_{-0.91}^{+0.91}$ & $1.46_{-0.03}^{+0.03}$ & $0.81(60)$ & diskbb+gau + pow \\
\hline 2010-03-16 & 95409-01-10-04 & $12.04 \pm 0.10$ & $218.29_{-0.69}^{+0.70}$ & $1.48_{-0.02}^{+0.02}$ & $0.79(60)$ & diskbb+gau + pow \\
\hline 2010-03-20 & 95409-01-11-01 & $15.45 \pm 0.06$ & $268.48_{-1.14}^{+1.15}$ & $1.47_{-0.04}^{+0.03}$ & $0.69(60)$ & diskbb+gau + pow \\
\hline 2010-03-22 & 95409-01-11-02 & $15.45 \pm 0.06$ & $291.93_{-1.38}^{+1.38}$ & $1.53_{-0.03}^{+0.03}$ & $0.80(60)$ & diskbb + gau + pow \\
\hline 2010-03-24 & 95409-01-11-03 & $18.59 \pm 0.05$ & $329.61_{-1.37}^{+1.38}$ & $1.52_{-0.03}^{+0.03}$ & $1.00(60)$ & diskbb+gau+pow \\
\hline 2010-03-26 & 95409-01-12-00 & $21.88 \pm 0.10$ & $352.09_{-1.19}^{+1.20}$ & $1.53_{-0.03}^{+0.02}$ & $0.89(60)$ & diskbb + gau + pow \\
\hline 2010-03-30 & $95409-01-12-02$ & $21.88 \pm 0.10$ & $405.22_{-1.48}^{+1.51}$ & $1.58_{-0.02}^{+0.02}$ & $1.36(60)$ & diskbb + gau + pow \\
\hline 2010-03-31 & 95409-01-12-04 & $25.94 \pm 0.05$ & $414.09_{-1.40}^{+1.41}$ & $1.62_{-0.02}^{+0.02}$ & $0.90(60)$ & diskbb+gau + pow \\
\hline 2010-04-03 & 95409-01-13-00 & $21.11 \pm 0.15$ & $442.55_{-1.38}^{+1.40}$ & $1.61_{-0.02}^{+0.02}$ & $1.14(60)$ & diskbb+gau + pow \\
\hline 2010-04-05 & 95409-01-13-02 & $24.69 \pm 0.05$ & $467.72_{-1.45}^{+1.46}$ & $1.63_{-0.02}^{+0.02}$ & $1.23(60)$ & diskbb + gau + pow \\
\hline 2010-04-06 & 95409-01-13-05 & $23.90 \pm 0.06$ & $491.54_{-1.91}^{+1.92}$ & $1.63_{-0.03}^{+0.02}$ & $1.02(60)$ & diskbb+gau + pow \\
\hline 2011-02-12 & 96409-01-07-03 & $4.17 \pm 0.05$ & $29.68_{-0.31}^{+0.31}$ & $1.76_{-0.02}^{+0.02}$ & $1.04(64)$ & pow \\
\hline
\end{tabular}


TABLE 1 - Continued from previous page

\begin{tabular}{ccccccc}
\hline Date & Obs. Id & $\begin{array}{c}F_{\mathrm{R}} \\
\text { 8. } 9.0 \mathrm{GHz} \\
\mathrm{mJy}\end{array}$ & $\begin{array}{c}F_{\mathrm{X}}-9 \mathrm{keV} \\
10^{-11} \mathrm{erg} / \mathrm{s} / \mathrm{cm}^{2}\end{array}$ & $\Gamma$ & $\chi_{\nu}^{2}(\mathrm{dof})$ & Model $^{a}$ \\
\hline $2011-02-14$ & $96409-01-07-01$ & $4.17 \pm 0.05$ & $25.68_{-0.27}^{+0.27}$ & $1.71_{-0.02}^{+0.02}$ & $1.23(64)$ & pow \\
$2011-02-15$ & $96409-01-07-02$ & $3.87 \pm 0.05$ & $34.65_{-0.30}^{+0.30}$ & $1.84_{-0.02}^{+0.02}$ & $1.27(64)$ & pow \\
$2011-02-17$ & $96409-01-07-04$ & $3.98 \pm 0.10$ & $20.19_{-0.24}^{+0.24}$ & $1.64_{-0.03}^{+0.03}$ & $0.63(64)$ & pow \\
$2011-02-19$ & $96409-01-08-00$ & $3.98 \pm 0.10$ & $16.14_{-0.21}^{+0.21}$ & $1.70_{-0.03}^{+0.03}$ & $0.95(64)$ & pow \\
$2011-02-21$ & $96409-01-08-02$ & $3.84 \pm 0.05$ & $12.16_{-0.27}^{+0.27}$ & $1.69_{-0.05}^{+0.05}$ & $0.80(64)$ & pow \\
$2011-02-24$ & $96409-01-08-03$ & $2.95 \pm 0.05$ & $9.72_{-0.23}^{+0.23}$ & $1.70_{-0.05}^{+0.06}$ & $1.00(64)$ & pow \\
$2011-02-28$ & $96409-01-09-01$ & $2.42 \pm 0.08$ & $7.20_{-0.25}^{+0.25}$ & $1.68_{-0.08}^{+0.08}$ & $0.45(64)$ & pow \\
$2011-03-02$ & $96409-01-09-02$ & $1.64 \pm 0.05$ & $6.55_{-0.21}^{+0.21}$ & $1.70_{-0.07}^{+0.07}$ & $0.64(64)$ & pow \\
$2011-03-06$ & $96409-01-10-01$ & $1.26 \pm 0.10$ & $5.11_{-0.28}^{+0.28}$ & $1.83_{-0.13}^{+0.13}$ & $0.62(64)$ & pow \\
$2011-03-08$ & $96409-01-10-02$ & $1.26 \pm 0.10$ & $4.69_{-0.14}^{+0.14}$ & $1.78_{-0.07}^{+0.07}$ & $0.67(64)$ & pow \\
$2011-03-10$ & $96409-01-10-03$ & $1.38 \pm 0.08$ & $4.47_{-0.14}^{+0.14}$ & $1.71_{-0.07}^{+0.07}$ & $0.52(64)$ & pow \\
$2011-03-19$ & $96409-01-12-01$ & $0.74 \pm 0.04$ & $2.38_{-0.12}^{+0.12}$ & $1.88_{-0.11}^{+0.12}$ & $0.64(64)$ & pow \\
\hline
\end{tabular}

${ }^{a}$ an absorption model, phabs, was used in all fittings.

TABLE 2. Summary of data for H 1743-322.

\begin{tabular}{|c|c|c|c|c|c|c|c|}
\hline Date & Obs. Id & $\begin{array}{c}F_{\mathrm{R}} \\
8.5 \mathrm{GHz} \\
\mathrm{mJy}\end{array}$ & $\begin{array}{c}F_{\mathrm{X}} \\
3-9 \mathrm{keV} \\
10^{-11} \mathrm{erg} / \mathrm{s} / \mathrm{cm}^{2}\end{array}$ & $\Gamma$ & $\Gamma_{\mathrm{H}}$ & $\chi^{2}$ & $\operatorname{Model}^{a}$ \\
\hline 2003-04-01 & $80138-01-02-00$ & $6.45 \pm 0.12$ & $253_{-0.42}^{+0.41}$ & $1.56_{-0.01}^{+0.01}$ & $2.19_{-0.01}^{+0.01}$ & $1.70(88)$ & diskbb+gau+bknpow \\
\hline 2003-04-04 & 80138-01-03-00 & $21.81 \pm 0.13$ & $626_{-0.63}^{+0.63}$ & $2.27_{-0.01}^{+0.01}$ & $2.89_{-0.02}^{+0.02}$ & $1.08(88)$ & diskbb+gau+bknpow \\
\hline 2003-04-06 & 80138-01-04-00 & $19.43 \pm 0.16$ & $545_{-0.55}^{+0.55}$ & $2.23_{-0.04}^{+0.01}$ & $3.0_{-0.02}^{+0.02}$ & $0.93(87)$ & diskbb+gau + bknpow \\
\hline 2003-11-05 & $80137-01-32-00$ & $0.22 \pm 0.05$ & $12.09_{-0.12}^{+0.11}$ & $\ldots$ & $1.95_{-0.02}^{+0.02}$ & $0.96(88)$ & gau + pow \\
\hline 2004-11-01 & 90115-01-05-03 & $0.31 \pm 0.06$ & $2.59_{-0.13}^{+0.13}$ & $\ldots$ & $2.16_{-0.05}^{+0.05}$ & $0.83(88)$ & gau+pow \\
\hline 2008-01-29 & $93427-01-03-03$ & $0.44 \pm 0.09$ & $70.35_{-0.25}^{+0.25}$ & $\ldots$ & $2.05_{-0.06}^{+0.06}$ & $1.05(87)$ & diskbb+gau+pow \\
\hline 2008-02-02 & 93427-01-04-00 & $0.52 \pm 0.07$ & $50.19_{-0.19}^{+0.19}$ & $\ldots$ & $1.93_{-0.04}^{+0.04}$ & $0.81(87)$ & diskbb+gau+pow \\
\hline 2008-02-04 & $93427-01-04-02$ & $0.48 \pm 0.08$ & $36.85_{-0.16}^{+0.16}$ & $\ldots$ & $1.94_{-0.01}^{+0.01}$ & $1.23(89)$ & gau+pow \\
\hline 2008-02-06 & 93427-01-04-03 & $0.45 \pm 0.09$ & $30.79_{-0.14}^{+0.15}$ & $\ldots$ & $1.93_{-0.01}^{+0.01}$ & $1.00(89)$ & gau+pow \\
\hline 2008-02-08 & $93427-01-05-00$ & $0.56 \pm 0.05$ & $25.94_{-0.18}^{+0.18}$ & $\ldots$ & $1.91_{-0.02}^{+0.01}$ & $1.01(89)$ & gau+pow \\
\hline $2008-02-18$ & 93427-01-06-02 & $0.23 \pm 0.12$ & $3.82_{-0.18}^{+0.18}$ & $\ldots$ & $2.03_{-0.05}^{+0.05}$ & $0.82(89)$ & gau+pow \\
\hline $2008-02-20$ & 93427-01-06-03 & $0.21 \pm 0.05$ & $2.72_{-0.11}^{+0.11}$ & $\ldots$ & $2.14_{-0.04}^{+0.04}$ & $0.79(89)$ & gau+pow \\
\hline $2008-02-22$ & $93427-01-07-00$ & $0.23 \pm 0.06$ & $1.87_{-0.14}^{+0.14}$ & $\ldots$ & $2.13_{-0.05}^{+0.05}$ & $0.92(89)$ & gau+pow \\
\hline $2008-02-24$ & $93427-01-07-02$ & $0.31 \pm 0.05$ & $1.41_{-0.26}^{+0.26}$ & $\ldots$ & $2.30_{-0.13}^{+0.15}$ & $1.04(89)$ & gau+pow \\
\hline $2008-10-07$ & 93427-01-09-01 & $1.74 \pm 0.07$ & $116.60_{-0.36}^{+0.36}$ & $1.50_{-0.04}^{+0.04}$ & $2.40_{-0.19}^{+0.27}$ & $1.18(85)$ & diskbb+gau+bknpow \\
\hline 2008-10-08 & 93427-01-09-03 & $2.54 \pm 0.08$ & $116.62_{-0.36}^{+0.36}$ & $1.50_{-0.05}^{+0.04}$ & $2.15_{-0.11}^{+0.13}$ & $0.72(85)$ & diskbb+gau +bknpow \\
\hline 2008-10-09 & 93427-01-09-02 & $2.43 \pm 0.09$ & $120.42_{-0.47}^{+0.47}$ & $1.34_{-0.10}^{+0.11}$ & $1.91_{-0.06}^{+0.06}$ & $0.94(85)$ & diskbb+gau+bknpow \\
\hline 2008-10-11 & $93427-01-10-00$ & $2.38 \pm 0.11$ & $121.92_{-0.63}^{+0.63}$ & $1.50_{-0.06}^{+0.11}$ & $2.08_{-0.16}^{+0.23}$ & $1.16(85)$ & diskbb+gau+bknpow \\
\hline 2008-11-04 & $93427-01-13-06$ & $0.94 \pm 0.12$ & $68.22_{-0.29}^{+0.29}$ & $\ldots$ & $1.92_{-0.01}^{+0.01}$ & $1.42(91)$ & pow \\
\hline 2008-11-09 & 93427-01-14-02 & $0.94 \pm 0.08$ & $60.86_{-0.39}^{+0.39}$ & $\ldots$ & $1.83_{-0.01}^{+0.01}$ & $1.38(91)$ & pow \\
\hline 2009-05-29 & $94413-01-02-00$ & $2.24 \pm 0.03$ & $153.29_{-0.56}^{+0.57}$ & $1.51_{-0.06}^{+0.06}$ & $2.16_{-0.09}^{+0.12}$ & $1.15(85)$ & diskbb+gau+bknpow \\
\hline 2009-05-30 & 94413-01-02-01 & $2.73 \pm 0.10$ & $163.60_{-0.68}^{+0.68}$ & $1.43_{-0.01}^{+0.05}$ & $2.07_{-0.04}^{+0.05}$ & $1.29(85)$ & diskbb+gau + bknpow \\
\hline 2009-07-07 & 94413-01-07-01 & $0.59 \pm 0.06$ & $51.56_{-0.36}^{+0.36}$ & $\ldots$ & $2.07_{-0.02}^{+0.02}$ & $0.94(91)$ & pow \\
\hline 2009-07-09 & 94413-01-07-02 & $0.41 \pm 0.07$ & $46.94_{-0.36}^{+0.36}$ & $\ldots$ & $2.10_{-0.02}^{+0.02}$ & $1.27(91)$ & pow \\
\hline 2009-07-11 & $94413-01-08-02$ & $0.34 \pm 0.06$ & $38.85_{-0.41}^{+0.41}$ & $\ldots$ & $2.02_{-0.02}^{+0.02}$ & $0.89(91)$ & pow \\
\hline 2009-07-13 & $94413-01-08-00$ & $0.59 \pm 0.07$ & $30.82_{-0.21}^{+0.21}$ & $\ldots$ & $1.97_{-0.01}^{+0.01}$ & $1.09(89)$ & gau+pow \\
\hline 2009-07-19 & 94413-01-09-00 & $0.63 \pm 0.05$ & $13.95_{-0.22}^{+0.22}$ & $\ldots$ & $1.87_{-0.03}^{+0.03}$ & $1.02(89)$ & gau+pow \\
\hline 2009-08-06 & 94413-01-11-02 & $0.19 \pm 0.05$ & $0.65_{-0.15}^{+0.15}$ & $\ldots$ & $2.25_{-0.08}^{+0.08}$ & $0.88(89)$ & gau+pow \\
\hline
\end{tabular}


Cao et al.

TABLE 3. Summary of data for Swift J1753.5-0127.

\begin{tabular}{|c|c|c|c|c|c|c|c|}
\hline Date & Obs. Id & $\begin{array}{c}F_{\mathrm{R}} \\
8.4 \mathrm{GHz} \\
\mathrm{mJy}\end{array}$ & $\begin{array}{c}F_{\mathrm{X}} \\
3-9 \mathrm{keV} \\
10^{-11} \mathrm{erg} / \mathrm{s} / \mathrm{cm}^{2}\end{array}$ & $\bar{\Gamma}$ & $\overline{\Gamma_{\mathrm{H}}}$ & $\chi^{2}$ & Model $^{a}$ \\
\hline 2005-07-04 & 91094-01-01-01 & $0.68 \pm 0.20$ & $300.44_{-0.68}^{+0.67}$ & $1.69_{-0.02}^{+0.02}$ & $2.22_{-0.09}^{+0.11}$ & $1.15(84)$ & diskbb+gau+bknpow \\
\hline 2005-07-06 & 91423-01-01-04 & $0.74 \pm 0.20$ & $342.78_{-0.74}^{+0.75}$ & $1.71_{-0.02}^{+0.02}$ & $2.07_{-0.06}^{+0.08}$ & $1.12(84)$ & diskbb+gau+bknpow \\
\hline 2005-07-07 & 91423-01-01-00 & $0.68 \pm 0.68$ & $315.09_{-0.82}^{+0.83}$ & $1.64_{-0.01}^{+0.01}$ & $1.96_{-0.03}^{+0.04}$ & $1.15(84)$ & diskbb+gau+bknpow \\
\hline $2005-07-08$ & 91094-01-02-01 & $1.96 \pm 0.04$ & $327.10_{-0.74}^{+0.75}$ & $1.66_{-0.02}^{+0.02}$ & $1.96_{-0.05}^{+0.07}$ & $1.03(84)$ & diskbb+gau+bknpow \\
\hline 2005-07-10 & 91094-01-02-02 & $1.14 \pm 0.12$ & $318.24_{-0.70}^{+0.70}$ & $1.63_{-0.02}^{+0.02}$ & $1.99_{-0.05}^{+0.06}$ & $0.86(84)$ & diskbb+gau+bknpow \\
\hline 2005-07-19 & 91423-01-03-04 & $2.42 \pm 0.05$ & $234.77_{-0.56}^{+0.56}$ & $1.59_{-0.02}^{+0.02}$ & $2.01_{-0.09}^{+0.13}$ & $0.95(84)$ & diskbb+gau+bknpow \\
\hline $2005-07-26$ & 91423-01-04-04 & $1.81 \pm 0.09$ & $186.15_{-0.47}^{+0.47}$ & $1.56_{-0.02}^{+0.02}$ & $2.24_{-0.16}^{+0.19}$ & $1.13(85)$ & diskbb+gau+bknpow \\
\hline 2005-08-03 & 91423-01-05-02 & $0.47 \pm 0.10$ & $141.72_{-0.39}^{+0.39}$ & $1.55_{-0.02}^{+0.02}$ & $1.83_{-0.09}^{+0.15}$ & $1.01(85)$ & diskbb+gau+bknpow \\
\hline 2005-08-07 & 91423-01-06-01 & $0.57 \pm 0.07$ & $118.55_{-0.36}^{+0.36}$ & $\ldots$ & $1.60_{-0.01}^{+0.01}$ & $1.22(91)$ & pow \\
\hline 2005-08-11 & 91423-01-06-03 & $0.68 \pm 0.01$ & $109.05_{-0.34}^{+0.33}$ & $\ldots$ & $1.61_{-0.01}^{+0.01}$ & $1.61(91)$ & pow \\
\hline $2005-10-22$ & 91423-01-17-00 & $0.28 \pm 0.06$ & $37.03_{-0.22}^{+0.22}$ & $\cdots$ & $1.60_{-0.01}^{+0.01}$ & $1.17(91)$ & pow \\
\hline 2005-11-19 & 91423-01-21-00 & $0.40 \pm 0.09$ & $30.40_{-0.22}^{+0.22}$ & $\ldots$ & $1.60_{-0.02}^{+0.02}$ & $0.78(91)$ & pow \\
\hline 2006-03-11 & 92404-01-02-00 & $0.08 \pm 0.07$ & $25.33_{-0.22}^{+0.22}$ & $\ldots$ & $1.65_{-0.02}^{+0.02}$ & $0.90(91)$ & pow \\
\hline 2007-07-01 & 93105-01-08-00 & $0.30 \pm 0.05$ & $45.52_{-0.20}^{+0.20}$ & $\ldots$ & $1.60_{-0.01}^{+0.01}$ & $1.09(91)$ & pow \\
\hline 2007-07-08 & 93105-01-09-00 & $0.30 \pm 0.05$ & $45.89_{-0.20}^{+0.20}$ & $\ldots$ & $1.61_{-0.01}^{+0.01}$ & $0.82(91)$ & pow \\
\hline 2007-07-15 & 93105-01-10-00 & $0.30 \pm 0.05$ & $46.01_{-0.20}^{+0.20}$ & $\ldots$ & $1.59_{-0.01}^{+0.01}$ & $1.03(91)$ & pow \\
\hline 2007-07-22 & 93105-01-11-00 & $0.08 \pm 0.08$ & $44.70_{-0.26}^{+0.25}$ & $\ldots$ & $1.60_{-0.01}^{+0.01}$ & $0.83(91)$ & pow \\
\hline 2009-06-09 & 93105-02-33-00 & $0.50 \pm 0.02$ & $50.95_{-0.22}^{+0.21}$ & $\ldots$ & $1.62_{-0.01}^{+0.01}$ & $1.09(91)$ & pow \\
\hline
\end{tabular}

$a^{a}$ an absorption model, phabs, was used in all fittings.

TABLE 4. Summary of data for XTE J1752-223.

\begin{tabular}{|c|c|c|c|c|c|c|c|}
\hline Date & Obs. Id & $\begin{array}{c}F_{\mathrm{R}} \\
8.4 \mathrm{GHz} \\
\mathrm{mJy} \\
\end{array}$ & $\begin{array}{c}F_{\mathrm{X}} \\
3-9 \mathrm{keV} \\
10^{-11} \mathrm{erg} / \mathrm{s} / \mathrm{cm}^{2}\end{array}$ & $\Gamma$ & $\Gamma_{\mathrm{H}}$ & $\chi^{2}$ & Model $^{a}$ \\
\hline $2009-10-30$ & 94331-01-02-01 & $2.42 \pm 0.05$ & $231.08_{-0.68}^{+0.68}$ & $1.38_{-0.02}^{+0.01}$ & $0.91(60)$ & diskbb+gau+pow & \\
\hline 2009-11-01 & 94331-01-02-05 & $1.99 \pm 0.08$ & $231.37_{-0.69}^{+0.69}$ & $1.37_{-0.02}^{+0.02}$ & $0.61(60)$ & diskbb + gau + pow & \\
\hline 2009-11-05 & 94331-01-02-17 & $2.05 \pm 0.07$ & $232.15_{-0.86}^{+0.87}$ & $1.38_{-0.02}^{+0.02}$ & $0.93(60)$ & diskbb+gau+pow & \\
\hline 2009-11-08 & 94331-01-03-05 & $2.10 \pm 0.10$ & $228.82_{-0.71}^{+0.71}$ & $1.39_{-0.02}^{+0.02}$ & $0.86(60)$ & diskbb+gau + pow & \\
\hline 2010-04-13 & 95360-01-12-03 & $0.90 \pm 0.05$ & $54.57_{-0.41}^{+0.41}$ & $1.77_{-0.02}^{+0.02}$ & $1.25(64)$ & pow & \\
\hline 2010-04-15 & $95360-01-12-04$ & $0.90 \pm 0.05$ & $52.05_{-0.49}^{+0.49}$ & $1.78_{-0.02}^{+0.02}$ & $1.23(64)$ & pow & \\
\hline 2010-04-17 & 95702-01-01-01 & $0.83 \pm 0.06$ & $48.92_{-0.39}^{+0.39}$ & $1.78_{-0.02}^{+0.02}$ & $1.23(64)$ & pow & \\
\hline 2010-04-27 & 95702-01-02-04 & $1.07 \pm 0.04$ & $33.59_{-0.32}^{+0.32}$ & $1.75_{-0.02}^{+0.02}$ & $0.84(64)$ & pow & \\
\hline 2010-04-28 & 95702-01-02-05 & $1.20 \pm 0.03$ & $31.88_{-0.31}^{+0.31}$ & $1.77_{-0.02}^{+0.02}$ & $1.08(64)$ & pow & \\
\hline 2010-04-29 & $95702-01-02-06$ & $1.13 \pm 0.04$ & $30.95_{-0.41}^{+0.41}$ & $1.74_{-0.03}^{+0.03}$ & $1.06(64)$ & pow & \\
\hline 2010-05-17 & 95702-01-05-03 & $0.28 \pm 0.04$ & $9.59_{-0.19}^{+0.19}$ & $1.77_{-0.05}^{+0.05}$ & $0.83(64)$ & pow & \\
\hline $2010-05-18$ & 95702-01-05-04 & $0.19 \pm 0.04$ & $9.26_{-0.24}^{+0.24}$ & $1.78_{-0.06}^{+0.06}$ & $0.84(64)$ & pow & \\
\hline 2010-06-03 & 95702-01-07-03 & $0.18 \pm 0.03$ & $12.78_{-0.28}^{+0.28}$ & $1.72_{-0.05}^{+0.05}$ & $0.82(64)$ & pow & \\
\hline 2010-06-11 & 95702-01-09-00 & $0.15 \pm 0.06$ & $15.81_{-0.31}^{+0.31}$ & $1.71_{-0.04}^{+0.04}$ & $0.77(64)$ & pow & \\
\hline
\end{tabular}

${ }^{a}$ an absorption model, phabs, was used in all fittings.

TABLE 5. Summary of the radio-X-ray fits.

\begin{tabular}{ll|cc|cc}
\hline Source & $F_{\text {crit, }}{ }^{a}$ & $k_{1}{ }^{b}$ & $b_{1}$ & $k_{2}{ }^{c}$ & $b_{2}$ \\
\hline GX 339-4 & $1.3_{-0.5}^{+0.7} \times 10^{-9}$ & $6.39 \pm 0.30$ & $0.61 \pm 0.03$ & $10.93 \pm 2.39$ & $1.14 \pm 0.27$ \\
H1743-322 & $2.5_{-0.5}^{+0.6} \times 10^{-10}$ & $1.91 \pm 1.04$ & $0.24 \pm 0.10$ & $12.83 \pm 1.29$ & $1.40 \pm 0.14$ \\
Swift J1753.5-0127 & $\lesssim 2.5 \times 10^{-10}$ & $\ldots$ & $\ldots$ & $9.55 \pm 1.23$ & $1.10 \pm 0.13$ \\
XTE J1752-223 & $1.6 \times \times_{-0.3}^{+0.2} 10^{-10}$ & $\ldots$ & $\ldots$ & $12.83 \pm 1.39$ & $1.39 \pm 0.19$ \\
\hline
\end{tabular}

$\bar{a}$ The critical flux for the anti- and positive correlations of $\Gamma-F_{3-9 \mathrm{keV}}$, and $1-\sigma$ errors are also included.

$b, c$ The best fits for the data points with anti- and positive correlations of $\Gamma-F_{3}-9 \mathrm{keV}$ with

a function of $F_{\mathrm{R}}=k F_{\mathrm{X}}^{b}$ respectively. 